University of Wollongong

Research Online

Faculty of Engineering and Information

Faculty of Engineering and Information

Sciences - Papers: Part B

Sciences

2018

\title{
A New Concentration Detection System for SF6/N2 Mixture Gas in Extra/ Ultra High Voltage Power Transmission Systems
}

Baojun Qu

Hebei University of Technology

Qingxin Yang

Tianjin Polytechnic University

Yongjian Li

Hebei University of Technology

Reza Malekian

University of Pretoria

Zhixiong $\mathrm{Li}$

China University of Mining and Technology, University of Wollongong

Follow this and additional works at: https://ro.uow.edu.au/eispapers1

Part of the Engineering Commons, and the Science and Technology Studies Commons

Research Online is the open access institutional repository for the University of Wollongong. For further information contact the UOW Library: research-pubs@uow.edu.au 


\title{
A New Concentration Detection System for SF6/N2 Mixture Gas in Extra/Ultra High Voltage Power Transmission Systems
}

\author{
Abstract \\ This paper develops a new detection system, for the first time, to detect the gas concentrations of the \\ SF6/N2 mixture in extra/ultra-high voltage power transmission systems. The concentrations of SF6 and \\ $\mathrm{N} 2$ are calculated from the thermal conductivity function of the mixture gas. The main contribution of this \\ work is that a specially-designed thermostatic chamber with adaptive temperature controller is developed \\ to ensure constant pressure of the gas flowed through the thermal conductivity sensor. Another \\ contribution is the combination of multiple sensors (e.g. humidity and electrochemical sensors), which \\ enables the detector to address the penetration effects of $\mathrm{H} 2 \mathrm{O}$ and $\mathrm{O} 2$ in the SF6/N2 mixture. \\ Experimental evaluation results using the prototype demonstrated that satisfactory accuracy ( \& \\ \#x00B1;1 \& \#x0025; of the measurement error) has been achieved for the concentration detection of the \\ SF6/N2 mixture under variable operation conditions. Compared with existing detection techniques, the \\ proposed detector not only can detect the SF6/N2 concentration by taking the air infiltration effect into \\ account, but also reduce the cost.

\section{Disciplines} \\ Engineering | Science and Technology Studies

\section{Publication Details} \\ Qu, B., Yang, Q., Li, Y., Malekian, R. \& Li, Z. (2018). A New Concentration Detection System for SF6/N2 \\ Mixture Gas in Extra/Ultra High Voltage Power Transmission Systems. IEEE Sensors Journal, 18 (9), \\ 3806-3812.
}




\title{
A New Concentration Detection System for $\mathrm{SF}_{6} / \mathrm{N}_{2}$ Mixture Gas in Extra/Ultra High Voltage Power Transmission Systems
}

\author{
Baojun Qu, Qingxin Yang, Yongjian Li, Member, IEEE, Reza Malekian, Senior Member, IEEE, \\ Zhixiong Li, Member, IEEE
}

\begin{abstract}
This paper develops a new detection system, for the first time, to detect the gas concentrations of the $\mathrm{SF}_{6} / \mathrm{N}_{2}$ mixture in extra/ultra-high voltage power transmission systems. The concentrations of $\mathrm{SF}_{6}$ and $\mathrm{N}_{2}$ are calculated from the thermal conductivity function of the mixture gas. The main contribution of this work is that a specially-designed thermostatic chamber with adaptive temperature controller is developed to ensure constant pressure of the gas flowed through the thermal conductivity sensor. Another contribution is the combination of multiple sensors (e.g. humidity and electrochemical sensors), which enables the detector to address the penetration effects of $\mathrm{H}_{2} \mathrm{O}$ and $\mathrm{O}_{2}$ in the $\mathrm{SF}_{6} / \mathrm{N}_{2}$ mixture. Experimental evaluation results using the prototype demonstrated that satisfactory accuracy $( \pm 1 \%$ of the measurement error) has been achieved for the concentration detection of the $\mathrm{SF}_{6} / \mathrm{N}_{2}$ mixture under variable operation conditions. Compared with existing detection techniques, the proposed detector not only can detect the $\mathrm{SF}_{6} / \mathrm{N}_{2}$ concentration by taking the air infiltration effect into account, but also reduce the cost.
\end{abstract}

Index Terms $-\mathrm{SF}_{6} / \mathbf{N}_{2}$, gas mixture, microfluidic thermal conductivity sensor, constant temperature control, trace $\mathrm{O}_{2}$

This project is support by the National Science Foundation of China (NSFC) (No. 51505265, 51505475 and U1610109), UOW VC Postdoctoral Fellowship, the National Research Foundation of South Africa (grant numbers: IFR160118156967 and RDYR160404161474) and Tertiary Education Support Programme (TESP), ESKOM, South Africa

Baojun Qu is with the Province-Ministry Joint Key Laboratory of EFEAR, Hebei University of Technology, Tianjin 300130, China. (e-mail: 529435754@qq.com).

Dr. Qingxin Yang is a full professor at Tianjin Key Laboratory of AEEET, Tianjin Polytechnic University, Tianjin 300387, China (e-mail: yangqingxin@tjpu.edu.cn).

Dr. Yongjian $\mathrm{Li}$ is a full professor at the Province-Ministry Joint Key Laboratory of EFEAR, Hebei University of Technology, Tianjin 300130, China. (e-mail: liyongjian@hebut.edu.cn).

Dr. Reza Malekian is with the Department of Electrical, Electronic and Computer Engineering, University of Pretoria, Pretoria 0002, South Africa (e-mail: reza.malekian@ieee.org).

Dr. Zhixiong Li is with the School of Mechatronic Engineering, China University of Mining and Technology, Xuzhou 221116, China; School of Mechanical, Materials, Mechatronic and Biomedical Engineering, University of Wollongong, Wollongong, NSW 2522, Australia (Corresponding author, e-mail: zhixiong.li@ieee.org).

\section{INTRODUCTION}

Owing to outstanding insulating characteristics and arc-quenching capacity, the Sulfur hexafluoride $\left(\mathrm{SF}_{6}\right)$ gas has been widely used in extra/ultra-high voltage power transmission systems [1-5]. Usually, $\mathrm{SF}_{6}$ gas is pressurized [6] in the electrical equipment to improve its capacitance and insulation performance because the heat capacity and dielectric strength increase with the pressure increase of $\mathrm{SF}_{6}$. However, with the increase of the gas pressure, the liquefaction temperature of $\mathrm{SF}_{6}$ will increase accordingly. Under the pressure of $0.22 \mathrm{MPa}$ (i.e., $275 \mathrm{kV}$ level), its liquefaction temperature is $-40^{\circ} \mathrm{C}$, whereas under $0.6 \mathrm{MPa}$ (i.e., $500 \mathrm{kV}$ level), the liquefaction temperature increases to $-25^{\circ} \mathrm{C}$ [7]. In cold regions, the liquefaction of the $\mathrm{SF}_{6}$ gas will cause the decrease of the $\mathrm{SF}_{6}$ pressure inside the electrical equipment, resulting in degradation of insulation performance [8]. In addition, $\mathrm{SF}_{6}$ gas is very costly and sensitive to non-uniform electric field [9]. The contribution of greenhouse effect of $\mathrm{SF}_{6}$ is 23,900 times of $\mathrm{CO}_{2}[10,11]$. More importantly, $\mathrm{SF}_{6}$ has been included in the gas list by the Kyoto Protocol (1997), whose emissions should be limited [12]. Hence, it is crucial to develop alternatives to $\mathrm{SF}_{6}$ in electrical equipment $[13,14]$.

Currently, the development of environmental-friendly replacement of $\mathrm{SF}_{6}$ is a hot topic, aiming to improve the insulation properties and reduce greenhouse effect of the gas in electrical equipment [15-18]. The mixture of $\mathrm{SF}_{6} / \mathrm{N}_{2}$ is an promising alternative to $\mathrm{SF}_{6}$ and has shown good potentials for practical applications $[19,20]$. For example, when the content of $\mathrm{SF}_{6}$ is $50 \%$ in the $\mathrm{SF}_{6} / \mathrm{N}_{2}$ mixture in uniform electric field, the electric strength of the mixed gas is almost $85 \%$ of the strength of the pure $\mathrm{SF}_{6}$ gas [17]. In addition, the cost of $\mathrm{SF}_{6} / \mathrm{N}_{2}$ is much cheaper and the emission is much smaller than that of $\mathrm{SF}_{6}$ while the stability and safety levels of $\mathrm{SF}_{6} / \mathrm{N}_{2}$ are much higher than that of $\mathrm{SF}_{6}$. The liquefaction problem of SF6 is also solved by the mixture of SF6/N2 (e.g., the liquefaction point of $60 \% \mathrm{SF} 6+40 \% \mathrm{~N} 2$ mixture is $-42^{\circ} \mathrm{C}$ under $0.6 \mathrm{MPa}$ ). As a result, the $\mathrm{SF}_{6} / \mathrm{N}_{2}$ mixture gas is gradually replacing the $\mathrm{SF}_{6}$ gas in extra/ultra-high voltage transmission systems [21-23]. For 
example, the insulation performance of $\mathrm{SF}_{6} / \mathrm{N}_{2}$ has already been evaluated in a real world $550 \mathrm{kV}$ power transmission system by the New Northeast Electric Company [22]. For another example, in more than $200 \mathrm{~km}$ of GIL (gas-insulated transmission line) installed by Alstom Grid, the $\mathrm{SF}_{6} / \mathrm{N}_{2}$ mixture has been considered in insulation design [13].

However, in practice $\mathrm{H}_{2} \mathrm{O}$ and $\mathrm{O}_{2}$ may penetrate into the $\mathrm{SF}_{6} / \mathrm{N}_{2}$ mixture during its service life, resulting in insulation performance degradation. For instance, the seal device may deteriorate after some usage time and $\mathrm{H}_{2} \mathrm{O}$ and $\mathrm{O}_{2}$ in the air may penetrate through the seal into the $\mathrm{SF}_{6} / \mathrm{N}_{2}$ mixture. It is crucial to detect the concentration of $\mathrm{H}_{2} \mathrm{O}$ and $\mathrm{O}_{2}$ in practice while an effective detection system is not developed in literature. Most of existing systems are developed for $\mathrm{SF}_{6}$ detection [24]. Generally, there are thermal conductivity sensor based [25], Infrared sensor based [26], ultrasonic sensor based [27], and photo-acoustic sensor based $[2,28] \mathrm{SF}_{6}$ gas detection systems. Huang et al. [22] used the thermal conductivity sensor to detect the $\mathrm{SF}_{6}$ concentration in electrical equipment. Wang et al. [26] combined the infrared and electrochemical $\mathrm{SO}_{2}$ sensors to detect the SF6 decomposition. Stone [27] used the ultrasonic sensor for partial discharge diagnostics in electrical equipment. Sherstov et al. [28] adopted the photo-acoustic sensor for $\mathrm{SF}_{6}$ detection. However, to the best of our knowledge, the detection of $\mathrm{SF}_{6} / \mathrm{N}_{2}$ mixture considering the $\mathrm{H}_{2} \mathrm{O}$ and $\mathrm{O}_{2}$ elements has not been found yet in literature [29-31].

In order to address the aforementioned issue, this paper aims to develop a new detection system for concentration detection of $\mathrm{SF}_{6} / \mathrm{N}_{2}$ mixture considering the $\mathrm{H}_{2} \mathrm{O}$ and $\mathrm{O}_{2}$ penetration effect in extra/ultra-high voltage power transmission systems. The working principle and detailed hardware construction were described in this paper. Particularly, the humidity and micro-oxygen sensors were integrated into the detection system to detect the $\mathrm{H}_{2} \mathrm{O}$ and $\mathrm{O}_{2}$ concentration in the $\mathrm{SF}_{6} / \mathrm{N}_{2}$ mixture. A temperature control circuit and specially-designed thermal conductivity room were developed to make accurate detection results of the $\mathrm{SF}_{6}$ and $\mathrm{N}_{2}$ concentrations. The contribution/novelty of this work include

1) the developed system is applicable and effective to detecting $\mathrm{SF}_{6} / \mathrm{N}_{2}$ mixture with $\mathrm{H}_{2} \mathrm{O}$ and $\mathrm{O}_{2}$ penetration effect;

2) for the first time, a prototype of the proposed detection system is developed.

Experimental tests under regular and extreme operating conditions were conducted to evaluate the performance of the prototype on $\mathrm{SF}_{6} / \mathrm{N}_{2}$ concentration detection.

\section{The Proposed DEtection System}

\section{A. System Design Principle}

Fig. 1 depicts the overall design of the $\mathrm{SF}_{6} / \mathrm{N}_{2}$ concentration detection system, which is mainly consisted of one thermostatic chamber temperature detection and control module, one signal detection and conditioning module, one moisture detection module, one oxygen detection and conditioning module, and one flow monitoring and conditioning module. The thermal detection module is installed in the thermal conduction chamber to ensure the consistency of the heat detection. The micro-thermal conductivity sensor TCS208F is used and its output is recorded by the signal detection and conditioning module. The moisture detection module adopts DMT242J sensor to detect $\mathrm{H}_{2} \mathrm{O}$ in the gas mixture and the oxygen detection and conditioning module measures the concentration of trace $\mathrm{O}_{2}$. The flow monitoring and conditioning module adopts the mass flow meter to measure the gas flow rate.

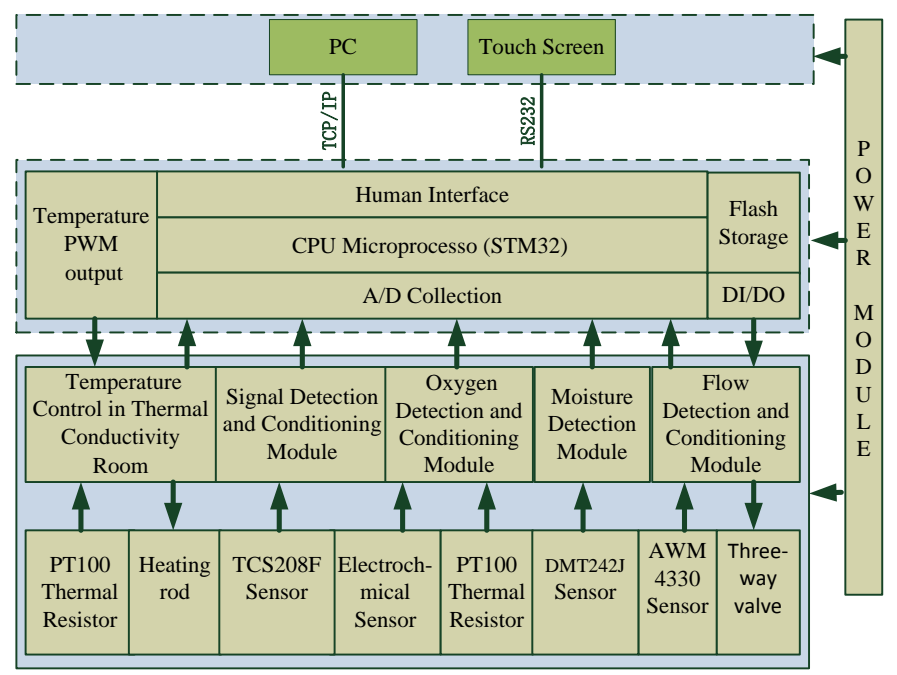

Fig. 1. The overall design of the $\mathrm{SF}_{6} / \mathrm{N}_{2}$ concentration detection system.

\section{B. Detection Principle of $\mathrm{SF}_{6} / \mathrm{N}_{2}$ Mixture}

Based on MEMS structure, the microfluidic thermal conductivity sensor (TCS208F) integrates metal thin-film thermosensitive elements, and in each element there are four resistors $\left(R_{m 1}, R_{m 2}, R_{t 1}\right.$ and $\left.R_{t 2}\right) . R_{m 1}$ and $R_{m 2}$ are used to heat the film and measure the film temperature. $R_{t 1}$ and $R_{t 2}$ are used to detect the ambient temperature and perform temperature compensation. Neglecting the effect of thermal convection and thermal radiation in the TCS208F sensor, the operation power of the film can be expressed as

$$
\begin{aligned}
P & =I_{\mathrm{m}}^{2} \cdot R_{\mathrm{m}} \cdot\left(1+\alpha \cdot T_{\mathrm{m}}\right) \\
& =I_{\mathrm{m}}{ }^{2} \cdot R_{\mathrm{m}} \cdot\left[1+\alpha \cdot\left(T_{\mathrm{m}}-T+T\right)\right]
\end{aligned}
$$

where $I_{m}$ is the sensor current, $R_{m}\left(=R_{m 1}+R_{m 2}\right)$ is the film resistor, $\alpha$ is the temperature coefficient, $T_{m}$ is the operation temperature, and $T$ is the initial ambient temperature.

Because the sensor heat is completely absorbed by the gas, the thermal equilibrium model in Eq. (1) can be simplified as 


$$
P=Q_{1}=\lambda \cdot S \cdot\left(T_{\mathrm{m}}-T\right) .
$$

Where $\lambda$ is the gas thermal conductivity and $S$ is the contact area between the film resistor and gas. The temperature difference $\Delta T$ after thermal equilibrium is

$$
\Delta T=T_{\mathrm{m}}-T=(1+\alpha T)\left(\frac{S \cdot \lambda}{I_{\mathrm{m}}^{2} R_{\mathrm{m}}}-\alpha\right)^{-1} .
$$

In order to ensure that the microfluidic thermal conductivity sensor works stably, a temperature control circuit is designed for the thermal conductivity sensor (see Fig. 2). In Fig. 2, the operational amplifier TLC2652 is used to amplify the micro signals. $R_{t 1}$ is controlled by the microcontroller to keep the temperature as a constant.

$$
\begin{aligned}
R_{\mathrm{t} 1} & =\frac{R_{1}}{R_{2}} \cdot\left(R_{\mathrm{m} 1}+R_{\mathrm{m} 2}\right) \cdot(1+\alpha \Delta T) \\
& =\frac{R_{1} \cdot \mathrm{R}_{\mathrm{m}}}{R_{2}} \cdot(1+\alpha \cdot \Delta T)
\end{aligned}
$$

where $R_{1}$ and $R_{2}$ are the resistors of the control circuit. Thus, the thermal conductivity of the mixture gas can be calculated by

$$
\lambda=\frac{\alpha \cdot U_{0}^{2}}{S} \cdot \frac{R_{\mathrm{m}} \cdot R_{\mathrm{t} 1}}{R_{\mathrm{t} 1} R_{2}^{2}-R_{\mathrm{m}} \cdot R_{1} \cdot R_{2}} .
$$

where $U_{0}$ is the sensor voltage. Eq. (5) is the thermal conductivity of $\mathrm{SF}_{6}, \mathrm{~N}_{2}$ and $\mathrm{O}_{2}$ mixture.

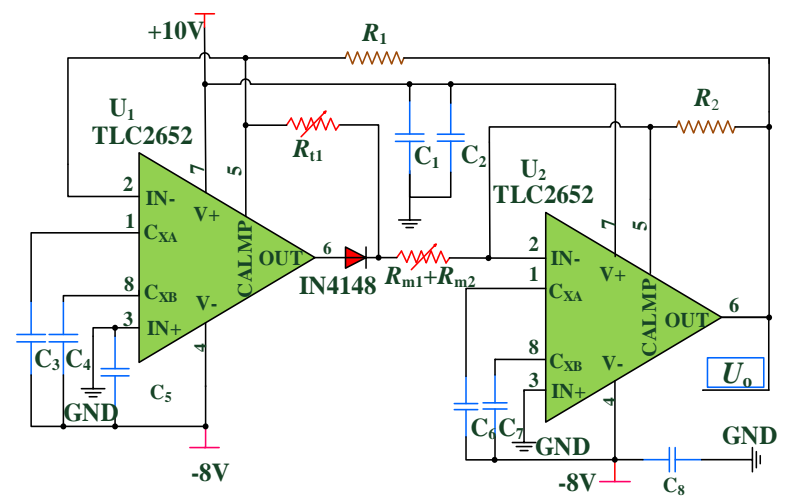

Fig. 2. The temperature control circuit of the thermal conductivity sensor.

When the system reaches the thermal equilibrium, $R_{m}$ and $R_{t 1}$ can be considered as constant values. As a result, a linear relationship can be learnt between $U_{0}$ and $\lambda$ from Eq. (5). Let us assume that the $\mathrm{O}_{2} / \mathrm{H}_{2} \mathrm{O}$ content infiltrated into the $\mathrm{SF}_{6} / \mathrm{N}_{2}$ mixtures meets the requirements of IEC60480-2004 [32]. So the chemical reactions in equilibrium condition can be expressed as follows:

$$
\begin{aligned}
& \mathrm{e}+\mathrm{SF}_{6} \rightarrow \mathrm{SF}_{\mathrm{n}}+(6-\mathrm{n}) \mathrm{F}+\mathrm{e}, \mathrm{n} \leqslant 5 \\
& \mathrm{SF}_{2}+\mathrm{O}_{2} \rightarrow \mathrm{SO}_{2} \mathrm{~F}_{2} \\
& \mathrm{e}+\mathrm{O}_{2} \rightarrow \mathrm{O}+\mathrm{O}+\mathrm{e} \\
& \mathrm{SF}_{5}+\mathrm{O} \rightarrow \mathrm{SOF}_{4}+\mathrm{F}
\end{aligned}
$$

Figure 3 depicts the equivalent diagram of the thermal conductivity sensor at its thermal equilibrium condition. It is used as a signal conditioning device to control the temperature of the gas.

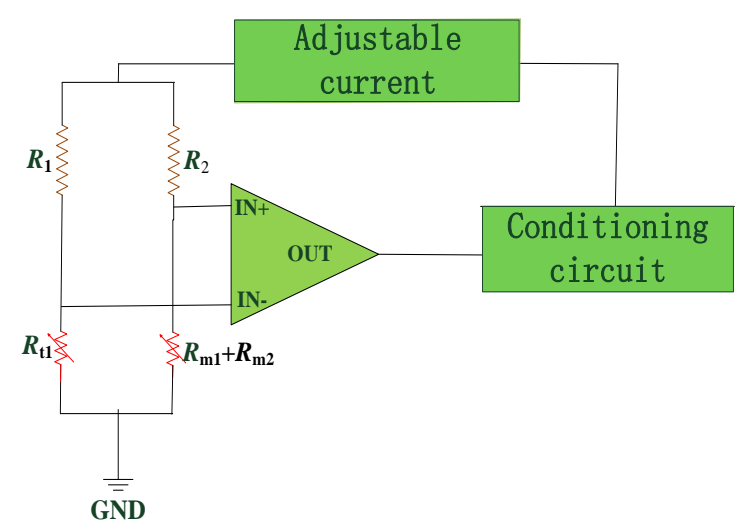

Fig. 3. The equivalent diagram of temperature control circuit in Fig. 2.

In Fig. 3, the Wheatstone bridge can be fall into balance condition through adjusting the resistances $R_{1}$ and $R_{2}$ after the passing of $\mathrm{SF}_{6} / \mathrm{N}_{2}$ mixture. The current determines the working temperature of the sensor at this moment. When the measured gas is passed through the sensor, the coefficient of thermal conductance $\lambda$ will increase, resulting in the decrease of the sensor temperature and the measured resistance $R_{m}$. As a result, the bridge is out of balance, and the unbalanced voltage is amplified and sent to the regulation circuit, where the output of the current source is adjusted so that the current is increased with the decrease of the sensor temperature. By doing so, no matter how the thermal conductivity $\lambda$ changes, the sensor temperature can keep as a constant to prevent inaccurate measurement or sensor damage due to overheating.

In order to determine the thermal conductivity of $\mathrm{SF}_{6} / \mathrm{N}_{2}$, it needs to know the thermal conductivity of $\mathrm{O}_{2}$. In the gas mixture of $\mathrm{SF}_{6}, \mathrm{~N}_{2}, \mathrm{H}_{2} \mathrm{O}$ and $\mathrm{O}_{2}$, due to the absence of the chemical reaction between the components, the thermal conductivity is approximated by the arithmetic mean of the thermal conductivity in Eq. (6).

$$
\lambda=\lambda_{1} C_{1}+\lambda_{2} C_{2}+\lambda_{3} C_{3}+\lambda_{4} C_{4}
$$

Where $\lambda$ denotes the thermal conductivity of the gas mixture, $\lambda_{i}$ $(i=1,2,3,4)$ respectively denotes the thermal conductivity of $\mathrm{SF}_{6}, \mathrm{~N}_{2}$ and $\mathrm{O}_{2}$, and $C_{i}(i=1,2,3,4)$ is the volume percentage of $\lambda_{i}$. The sum of volume percentage parameters satisfies 


$$
C_{1}+C_{2}+C_{3}+C_{4}=1
$$

From Eqs. (6) and (7) it can be seen that the concentrations of $\mathrm{SF}_{6}$ and $\mathrm{N}_{2}$ can be obtained as long as the $\mathrm{H}_{2} \mathrm{O}$ and $\mathrm{O}_{2}$ is detected.

\section{Detection of the Oxygen Concentration}

The micro-oxygen sensor is based on a lead-oxygen battery, including a lead anode, a gold cathode, and an alkaline electrolyte. Oxygen molecules enter into the electrochemical cell through a non-porous fluorine resin membrane and are reduced at the gold electrode. Without considering the temperature effect, the current generated by the Oxygen sensor is proportional to the oxygen concentration in the mixture gas [17]. When the membrane permeability is very low, the gas pressure is constant. This is because in the front end of the heat conduction chamber (see Section III) there is a long spiral tube buffer and a constant pressure device. The back-end of the heat conduction chamber is connected with the atmospheric pressure recovery device. If the external load resistance is small enough, the sensor output current can be expressed as

$$
i_{d}=k \cdot C_{p 3} .
$$

where, $i_{d}$ is the sensor output current, $k$ is the current concentration coefficient associated with the sensor structure and temperature, and $C_{p 3}$ is the measured oxygen concentration.

The output signal (i.e., $0-5 \mathrm{~V}$ voltage) is converted by the conditioning circuit in Fig. 4. The input of the conditioning circuit is the output signal of the oxygen sensor, which is conversed to the corresponding oxygen concentration by CPU.

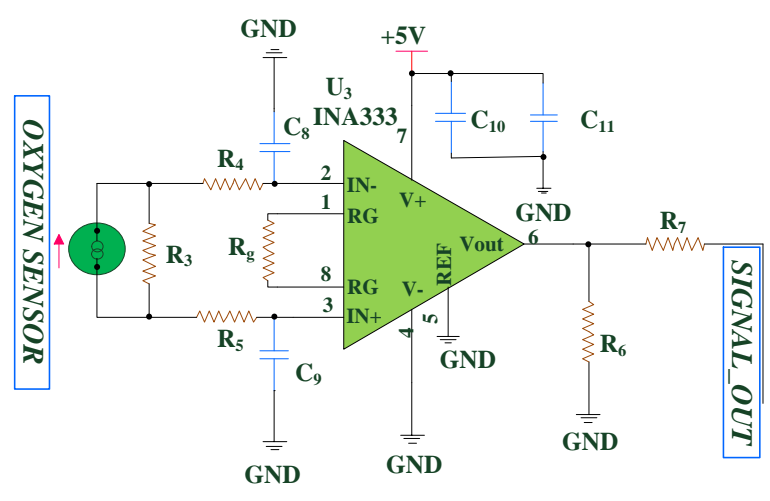

Fig. 4. Oxygen sensor conditioning circuit.

\section{Detection of Moisture Concentration}

The DMT242J moisture sensor and PT-100 thermal resistor are combined to measure the moisture in the mixture gas (see Fig. 1). DMT242J is usually used for reliable dew-point measurement in dry processes (such as industrial dryer applications). It mainly consists of a high polymer humicap and a thermistor. Its capacitance value is proportional to relative humidity $(R H)$ of the gas. PT-100 measures the temperature $(T)$ of the gas. According to the Goff-Gratch and Wexler correction formulae, the Saturation Vapour Pressure $P_{w s}$ at current temperature $T$ can be obtained. So the vapour pressure $P_{w}$ in the gas can be calculated by

$$
P_{w}=R H \cdot P_{w s}
$$

Then the absolute moisture amount can be calculated from $P_{w}$ using the specific parameters of DMT242.

\section{E. A Calculation Example}

In order to illustrate the process of the concentration detection of the proposed system, an example is carried out. The calculation process can be expressed as the following five steps.

Step 1: Calculate the thermal conductivity $\square$ of the gas mixture using Eq. (5)

Step 2: Measure the $\mathrm{O}_{2}$ and $\mathrm{H}_{2} \mathrm{O}$ concentration using the electrochemical and moisture sensors to obtain $C_{3}$ and $C_{4}$ in Eq. (6) at the current temperature $T$

Step 3: Find $\lambda_{i}(i=1,2,3,4)$ at temperature $T$

Step 4: Solve Eqs. (6) and (7) to get the results of $C_{1}$ and $C_{2}$ Step5: The concentrations of $\mathrm{SF}_{6}$ and $\mathrm{N}_{2}$ are respectively $C_{1}$ and $\mathrm{C}_{2}$

For example, given the temperature of $65^{\circ} \mathrm{C}$, the thermal conductivity of $\mathrm{SF}_{6}, \mathrm{~N}_{2}, \mathrm{H}_{2} \mathrm{O}$ and $\mathrm{O}_{2}$ are $\lambda_{1}=4.6 \mathrm{~W} / \mathrm{mK}, \lambda_{2}=$ $6.9 \mathrm{~W} / \mathrm{mK}, \lambda_{3}=4 \mathrm{~W} / \mathrm{mK}$ and $\lambda_{4}=7 \mathrm{~W} / \mathrm{mK}$. The thermal conductivity $\lambda$ of the gas mixture can be calculated by Eq. (5), i.e., $\lambda=5.5 \mathrm{~W} / \mathrm{mK}$. Then we measure the concentrations of $\mathrm{H}_{2} \mathrm{O}$ and $\mathrm{O}_{2}$ by the mixture and electrochemical sensors as $C_{3}=$ $0.1 \%$ and $C_{4}=0.6 \%$. Substituting these values into Eqs. (6) and (7) we can derive

$$
\left\{\begin{array}{c}
4.6 \times C_{1}+6.9 C_{2}+4 \times 0.1 \%+7 \times 0.6 \%=5.5 \\
C_{1}+C_{2}+0.1 \%+0.6 \%=1
\end{array} .\right.
$$

By solving Eq. (10) it yields $C_{1}=60.8 \%$ and $C_{2}=38.5 \%$, i.e., the concentrations of $\mathrm{SF}_{6}$ and $\mathrm{N}_{2}$ are respectively $60.8 \%$ and $38.5 \%$.

\section{Prototype of the Proposed Detection System}

\section{A. Design of Thermal Conductivity Room}

In order to ensure constant pressure of the gas flowed through the thermal conductivity sensor, a specially-designed thermostatic chamber is developed. The mechanical structure of the thermostatic chamber is shown in Fig. 5, where the inlet spiral pipeline functions as a buffer to stabilize the pressure of the gas flow. Then the gas is preheated by a helical heating rod, in which the temperature is controlled by the temperature conditioning circuit (see Fig. 2) to keep as a constant value for the chamber. Thanks to the buffer effect and heating function of 
the chamber, smooth gas pressure and constant temperature can be achieved to reduce/eliminate the measure error of the thermal conductivity sensor.

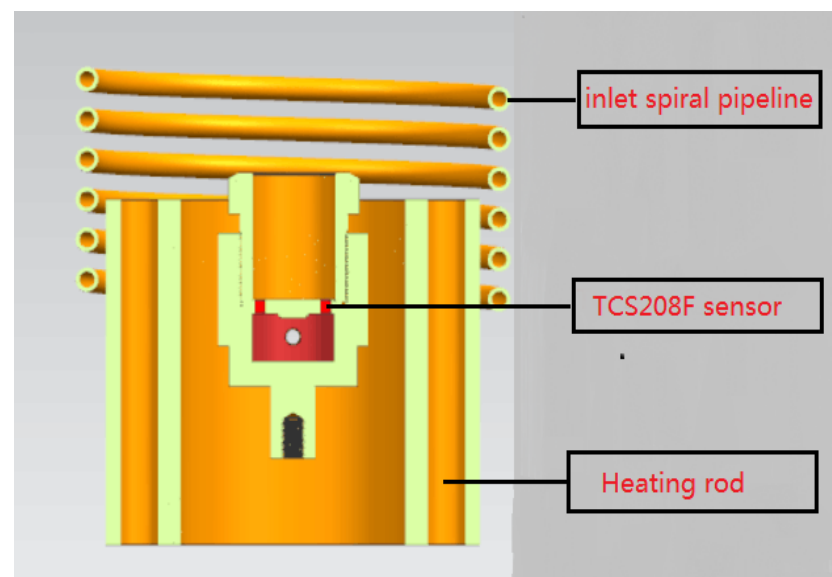

Fig. 5. Mechanical structure of the thermostatic chamber.

Here a simulation analysis was conducted to determine the optimum operating temperature of the thermal conductivity sensor. A simplified model of TCS208F microfluidic thermal conductivity sensor was established by FLUENT software. The flow-solid coupling field of the sensor model was simulated in a $20 \mathrm{~mL} / \mathrm{min}$ air flow-field. The analysis result is shown in Fig. 6. From the sensor temperature diagram in the figure it can be seen that, the central temperature of the sensor reaches to $336 \sim 343 \mathrm{~K}$ (i.e., $63 \sim 70^{\circ} \mathrm{C}$ ). Hence, it is generally recommended that the difference temperature $\Delta T$ between the sensor operation temperature and ambient temperature is within $50^{\circ} \mathrm{C}$, and the working temperature of the thermostatic chamber is within $75^{\circ} \mathrm{C}$.

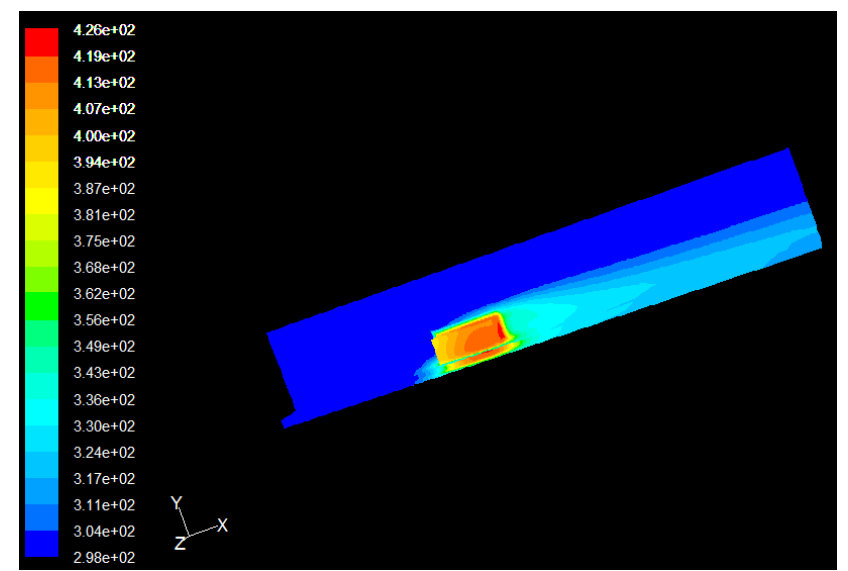

Fig. 6. Cloud picture around the TCS208F ambient temperature.

\section{B. Prototype of the Proposed Detection System}

The prototype of the proposed $\mathrm{SF}_{6} / \mathrm{N}_{2}$ concentration detector is shown in Fig. 7. When the temperature of the thermostatic chamber is stabilized at the setting value, the gas mixture enters into the thermostatic chamber. The TCS208F microfluidic thermal conductivity sensor then detects the $\mathrm{SF}_{6} / \mathrm{N}_{2}$ concentration. The detection result will be displayed in the screen and saved in the signal module.

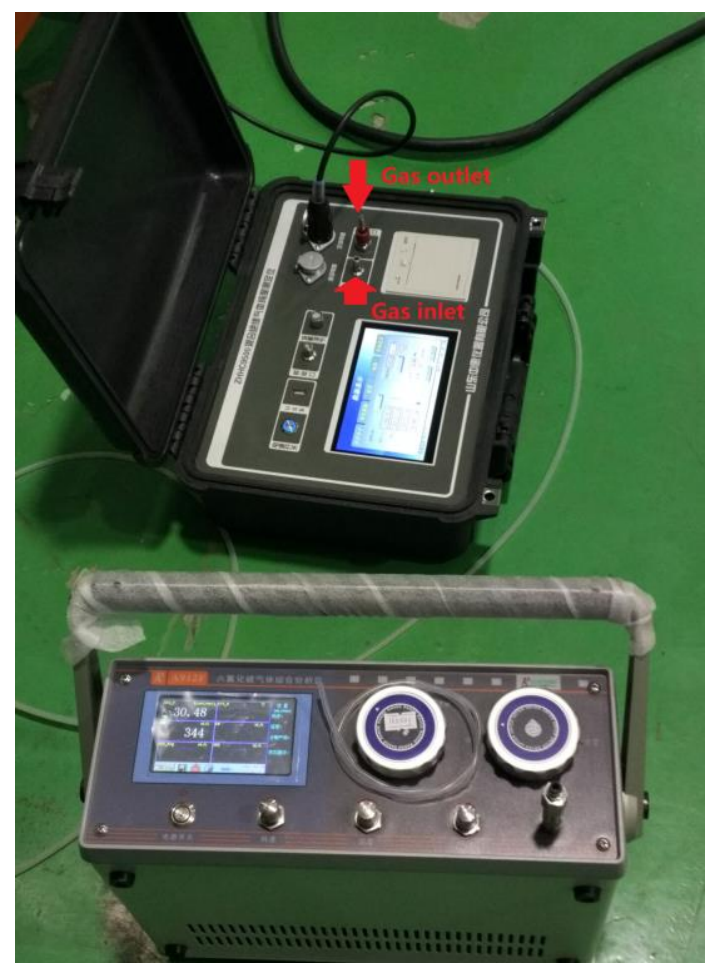

Fig. 7. The prototype of the proposed SF6/N2 concentration detector.

\section{RESUlts AND Discussion}

The detector prototype (see Fig. 7) was used to detect the mixture of $\mathrm{SF}_{6}, \mathrm{~N}_{2}, \mathrm{H}_{2} \mathrm{O}$ and $\mathrm{O}_{2}$ in the experimental test (see Fig. 8). According to CIGRE $[33,34]$, these four gases were diluted by a standard gas dilution device with a concentration of $32.19 \%\left(\mathrm{SF}_{6}\right), 67.2 \%\left(\mathrm{~N}_{2}\right), 0.01 \%\left(\mathrm{H}_{2} \mathrm{O}\right)$ and $0.6 \%\left(\mathrm{O}_{2}\right)$. Ten samples of the mixed gas were prepared and passed into the detector in the experiments. The temperature of the chamber was $22^{\circ} \mathrm{C} \pm 3^{\circ} \mathrm{C}$. The concentration of each gas element in each sample was repeatedly measured for 8 times under the stable operation condition of the detector, and the mean value of the measurements was taken as the final measurement for each sample. Fig. 9 manifests the detection results for the concentrations of the mixture gas using one sample. As can be seen in Fig. 9, the measured concentrations of the four gas elements were well consistent with the true values in the mixture. The mean square errors of the detection results in Fig. 9 were less than $0.05 \%$ for each element. Hence, the concentration detection accuracy of the prototype meets industrial criterion. 


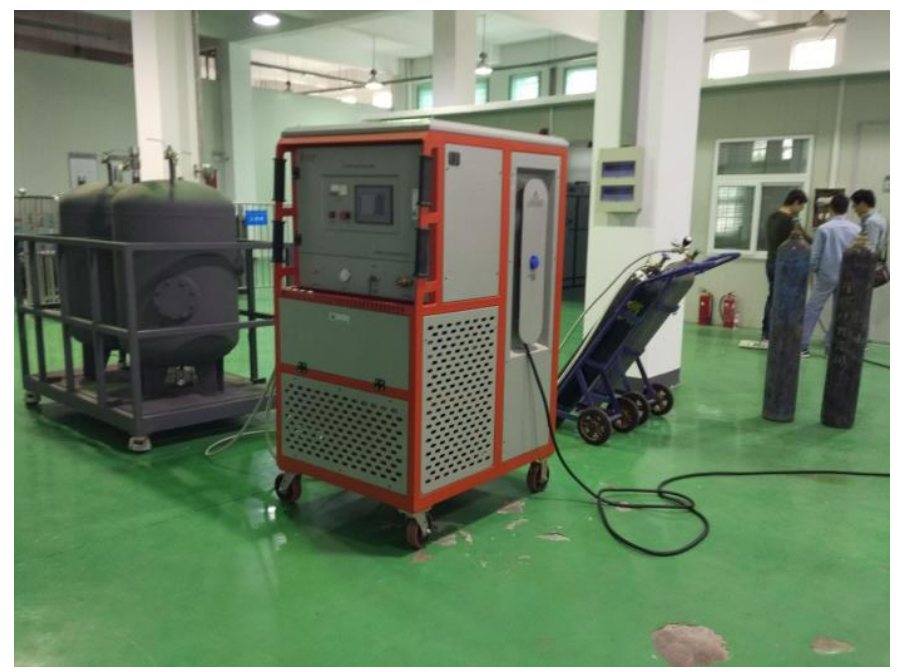

Fig. 8. The experimental tests using the proposed SF6/N2 concentration detection prototype.
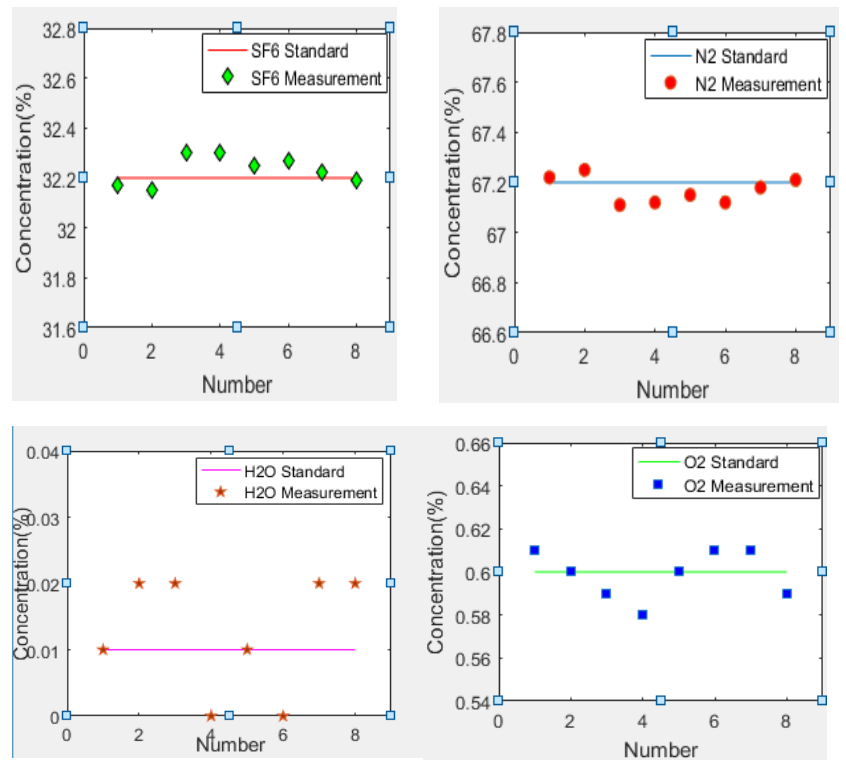

Fig. 9. The concentration detection results: (upper left) $\mathrm{SF}_{6}$, (upper right) $\mathrm{N}_{2}$, (lower left) $\mathrm{H}_{2} \mathrm{O}$, and (lower right) $\mathrm{O}_{2}$.

Furthermore, the detection performance of the prototype was evaluated under extreme temperature conditions of the chamber, that is, high temperature $\left(40^{\circ} \mathrm{C}+3^{\circ} \mathrm{C}\right)$ and low temperature $\left(-10^{\circ} \mathrm{C} \pm 3^{\circ} \mathrm{C}\right)$ conditions. Ten samples were prepared for each extreme condition. The repeatability error $C_{r}$ of the detection results was calculated by Eq. (11).

$$
C_{\mathrm{r}}=\frac{1}{\bar{N}}\left(\sum_{i=1}^{8}\left(N_{i}-\bar{N}\right)\right)^{\frac{1}{2}} \times 100 \%
$$

where, $N_{i}(i=1,2, \ldots, 8)$ is the $i$ th measurement of each element in one sample, and $\bar{N}$ is the mean value of the concentration of each gas for this sample. The concentration detection errors of the four elements in the gas mixture are shown in Fig. 10.
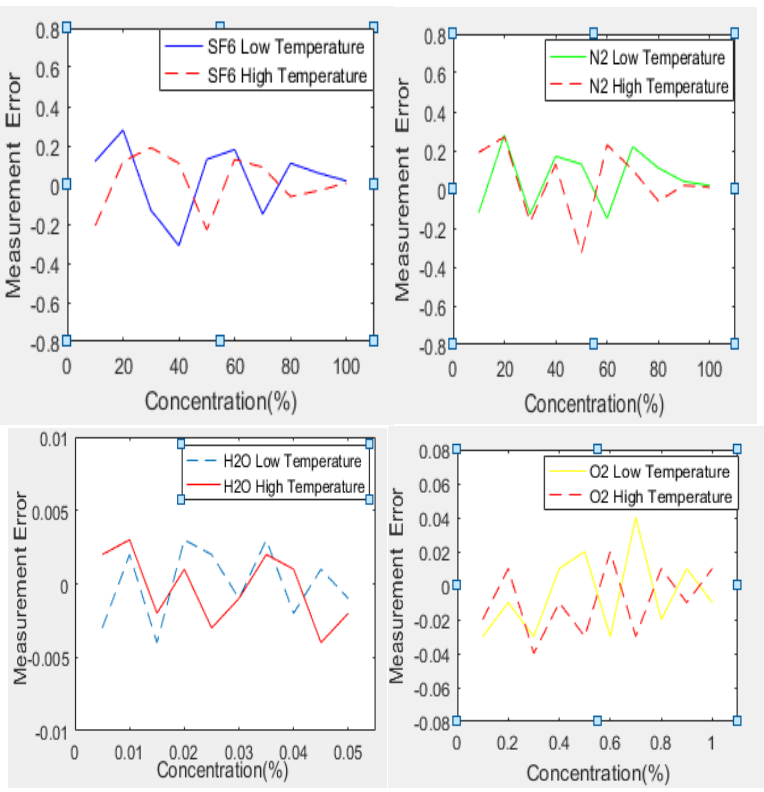

Fig. 10. The detection results under extreme temperature conditions: (upper left) $\mathrm{SF}_{6}$, (upper right) $\mathrm{N}_{2}$, (lower left) $\mathrm{H}_{2} \mathrm{O}$, and (lower right) $\mathrm{O}_{2}$.

As can be seen in Fig. 10, the detection errors of $\mathrm{SF}_{6}, \mathrm{~N}_{2}$, $\mathrm{H}_{2} \mathrm{O}$ and $\mathrm{O}_{2}$ for one sample were $0.41 \%, 0.43 \%, 0.002 \%$ and $0.04 \%$, respectively. The averaging repeated detection errors for all 10 samples were $\pm 0.21 \%, \pm 0.09 \%, \pm 1.4 \%$ and $\pm 1.3 \%$ for the four gas elements, respectively. According to the International ISO Standard 14040 [35], IEC60480-2004 [32] and National Standard of China, the maximum allowable error of the detector for $\mathrm{SF}_{6}, \mathrm{~N}_{2}, \mathrm{H}_{2} \mathrm{O}$ and $\mathrm{O}_{2}$ are respectively $\pm 0.5 \%$, $\pm 0.5 \%, \pm 0.05 \%$ and $\pm 0.05 \%$. The maximum allowable repeatability errors are $1 \%, 1 \%, 2 \%$ and $2 \%$. As a result, the detection accuracy and reliability of the prototype under extreme high and low temperature conditions meet the industrial requirements.

In this work the thermal conductivity sensor is adopted in the detector. This is because the thermal conductivity sensor based system is much cheaper than that of the infrared sensor or ultrasonic sensor or photo-acoustic sensor based systems. For instance, the price of thermal conductivity sensor based system is only $1 / 5$ of the infrared sensor system. In addition, the infrared sensors are often subject to temperature changes while the propose system has been proven to be robust to temperature. Furthermore, current international standards [32-34] require that the gas concentration is expressed as a mass concentration unit. Thanks to the specially-designed thermostatic chamber with temperature control unit, the measured volume concentration (ppm) of the proposed detection system can be 
easily converted into mass concentration (e.g., $\mathrm{mg} / \mathrm{m}^{3}$ ) while some other sensor measurements (e.g., infrared sensor and ultrasonic sensor) need to add pressure and temperature modules for the conversion, which may increase the cost and complexity of the detection system further. As a result, the thermal conductivity sensor is more suitable for the proposed detection system than other sensors in practical applications.

\section{CONCLUSION}

To address the industrial demand on accurate and reliable concentration detection of $\mathrm{SF}_{6} / \mathrm{N}_{2}$ mixture in extra/ultra-high voltage power transmission systems, this paper introduces a new system for the mixture concentration detection. The effect of $\mathrm{H}_{2} \mathrm{O}$ and $\mathrm{O}_{2}$ infiltration on the concentration of the mixture is solved by this new detector. The prototype of the proposed concentration detector for $\mathrm{SF}_{6} / \mathrm{N}_{2}$ mixture has been manufactured. The detection results of the prototype demonstrated that the measurement errors of $\mathrm{SF}_{6}$ and $\mathrm{N}_{2}$ were within $\pm 0.05 \%$, and their repeatability error was less than $1.0 \%$. The measurement errors of $\mathrm{H}_{2} \mathrm{O}$ and $\mathrm{O}_{2}$ were less than $0.04 \%$, and their repeatability errors were less than $2 \%$. According to the International ISO 14040 and IEC60480-2004 standards, these detection errors fulfil the industrial requirements. The proposed detector was also suitable and effective for $\mathrm{SF}_{6} / \mathrm{N}_{2}$ detection under extremely temperature conditions. As a result, the proposed detector is applicable to practical applications.

The reliability issue of the proposed detection system is worth investigating. In this work we only have tested its performance under $\left(40^{\circ} \mathrm{C} \pm 3^{\circ} \mathrm{C}\right)$ and $\left(-10^{\circ} \mathrm{C}+3^{\circ} \mathrm{C}\right)$. Because a thermostatic chamber is designed to stabilize the gas pressure and a temperature control circuit is used to obtain suitable operating temperature of the sensors, the detection system is expected to be safe under the tested extreme conditions in this work. In future we will comprehensively investigate the reliability issue of the proposed detection system using finite element analysis and experimental evaluation to discuss the influence factors to the system reliability under more severe operating conditions. In addition, future work will also compare the concentration detection performance of different sensors (e.g., infrared sensor or ultrasonic sensor or photo-acoustic sensor) using the sensor-array technique in the designed thermostatic chamber.

\section{REFERENCES}

[1] Y. Liu, W. Tao and K. Liu, "A spatially selective correlative filtration method based on the multiscale edge shift-correlative technique", IEEE Transactions on Instrumentation and Measurement, vol. 59, pp. 2280-2285, 2010.

[2] W. Cai, J. Tang, L. Cheng, C. Zhang, M. Fan, Q. Zhou and Q. Yao, "Detection of SF6 decomposition components under partial discharge by photoacoustic spectrometry and its temperature characteristic", IEEE Transactions on Instrumentation and Measurement, vol. 65, pp. 1343-1351, 2016.

[3] Z. Milanovic, K. Stankovic, M. Vujisic, R. Radosavljevic, and P. Osmokrovic, "Calculation of impulse characteristics for gas-insulated system with homogenous electric field," IEEE Trans. Dielectr. Electr. Insul., vol. 19, pp. 648-659, 2012.

[4] K. Stankovic, M. Pesic, P. Osmokrovic, and M. Vujisic, "Surface-time enlargement law for gas breakdown", IEEE Trans. Dielect. Electr. Insul., vol. 15, pp. 994-1005, 2008.

[5] S. Dekic, P. Osmokrovic, M. Vujisic, and K. Stankovic, "Conditions for the applicability of the geometrical similarity law to impulse breakdown in gases", IEEE Trans. Dielect. Electr. Insul., Vol. 17, pp. 1185-1195,2010.

[6] X. Zhang, B. Yang, W. Liu, J. Zhang, "Detection of partial discharge in SF6 decomposition gas based on modified carbon nanotubes sensors Jinbin", Procedia Engineering, vol. 29, pp. 4107-4111, 2012.

[7] X. Zhang, F. Meng, R. Li, Y. Liao, B. Yang, "Gas sensitivity studies on hydroxyl modified single-wall carbon nanotube detecting SF6 decomposed components under PD", High Voltage Engineering, vol. 39, pp. 1069-1074, 2013.

[8] P. Osmokrovic, "Electrical breakdown of SF6 at small values of the product pd", IEEE transactions on power delivery, vol. 4, pp. 2095-2099, 1989.

[9] L. Man, Y. Deng, D. Xiao, "Insulating Properties of c-C4F8/N2 and c-C4F8/CO2 Mixtures", High Voltage Engineering, vol. 43, pp. 788-794, 2017.

[10] Y. Qiu and E. Kuffel, "Comparison of SF6/N2 and SF6/CO2 gas mixtures as alternatives to SF6 gas", IEEE Trans Dielectrics and Electrical Insulation, vol. 6, pp. 892-895, 1996.

[11] C. Zhang, H. Shi, C. Lin, K. Zhao, "First Principles based computational scheme for designing new SF6 replacements", IEEE Transactions on Dielectrics and Electrical Insulation. vol. 23, pp. 2572-2578, 2016.

[12] Wartmann, Sina, and Jochen Harnisch. "Reductions of SF6 emissions from high and medium voltage electrical equipment in Europe." Environmental Sciences, vol. 2, pp. 273-281, 2005.

[13] Y. Kieffel, I. Todd, P. Philippe, O. John, "Green gas to replace SF6 in electrical grids." IEEE Power and Energy Magazine 14.2 (2016): 32-39.

[14] G. Xu, Q. Li, J. Zhang. "Study on SF6/CF4 as cooling and insulating medium of transformer", Journal of Tsinghua University (Science and Technology), vol. 37, pp. 16-19, 1997.

[15] W. Niu, J. Wei, D. Zhang and Y. Sun, "Insulation performance of $550 \mathrm{kV}$ SF6/N2 busbar", in Proceeding of 2012 Symposium on Annual Conference of Power Transmission and Transformation.

[16] H. Nechmi, A. Beroual, A. Girodet, P. Vinson, "Fluoronitriles/CO2 gas mixture as promising substitute to SF6 for insulation in high voltage applications", IEEE Transactions on Dielectrics and Electrical Insulation, vol. 23, pp. 2587-93, 2016.

[17] H. Zhao, X. Li, K. Zhu, Q. Wang, H. Lin, X. Guo, "Study of the arc interruption performance of SF6-CO2 mixtures as a substitute for SF6", IEEE Transactions on Dielectrics and Electrical Insulation, vol. 23, pp. 2657-2667, 2016.

[18] Y. Kieffel, "Characteristics of g3-an alternative to SF6." in Proceedings of 2016 IEEE International Conference on Dielectrics (ICD), 3-7 July 2016, Montpellier, France.

[19] Y. Kieffel, F. Biquez, P. Ponchon, "SF6 alternative development for high voltage switchgears", in Proceedings of 2015 IEEE Electrical Insulation Conference (EIC), 7-10 June 2015, Seattle, WA, USA.

[20] Y. Hoshina, M. Sato, H. Murase, M. Toyada, A. Kobayashi, "Dielectric properties of SF6/N2 gas mixtures on a full scale model of the gas-insulated busbar", in Proceedings of 2000 IEEE Power Engineering Society, vol. 3, pp. 2129-2134, 2000.

[21] F. Wang, X. Xiao, X. Xian, T. Fu, Q. Wang, G. Zhang. "Based on surface discharge characteristics study of insulator PIC method in SF6/N2 gas mixtures", Transactions of China Electrotechnical Society, vol. 26, pp. 220-226, 2011.

[22] C. Huang, J. Sun, X. Wang, S. He. "Analyzing system of SF6 gas in electrical equipment”, High Voltage Apparatus. vol.46, pp. 74-76, 2010.

[23] W. Liu, Z. Su, J. Qi, F. Zhu, Y. Zhao, F. Ma, X. Yuan and Y. Chen, "Application and recycling of SF6 gas mixtures in gas-insulated circuit breaker in northern China", IOP Conf. Ser.: Earth Environ. Sci., vol. 69, pp. 012002, 2017.

[24] X. Liu, S. Cheng, H. Liu, S. Hu, D. Zhang and H. Ning, "A survey on gas sensing technology", Sensors, vol. 12, pp. 9635-9665, 2012. 
[25] Z. Cai, L. Guerrero, A. Louwerse, H. Suy, R. van Veldhoven, K. Makinwa, M. Pertijs, "A CMOS readout circuit for resistive transducers based on algorithmic resistance and power measurement", IEEE Sensors Journal, vol. 17, no. 23, pp. 7917-7927, 2017.

[26] Q. Wang, C. Che, B. Yan, B. Chen, "Characteristics research of infrared and electrochemical SO2 sensor in SF6 decomposition detection", Instrument Technique and Sensor, vol. 6, pp. 7-10, 2016.

[27] G. Stone, "Partial discharge diagnostics and electrical equipment insulation condition assessment." IEEE Transactions on Dielectrics and Electrical Insulation, vol. 12, pp. 891-904, 2005.

[28] I. Sherstov, V. Vasiliev, K. Zenov, R. Pustovalova, V. Spitcin, S. Chernikov, "Development and research of a laser photo-acoustic SF6 gas analyzer", Instruments and Experimental Techniques, vol. 60, pp. 407-413, 2017.

[29] S. Kumar, T. Islam, K. K. Raina, "Modeling of breather for transformer health assessment", IET Science, Measurement \& Technology, vol. 11, no 2, pp. 194-203, 2017.

[30] L. Kumar, T. Islam, S. Mukhopadhyay, "Sensitivity enhancement of a PPM level capacitive moisture sensor." Electronics, vol. 6, no. 2, pp. 41, 2017.

[31] Yashdeep, G.R. Biswal, T. Choudhury, T. Islam, S.C. Mukhopadhyay, V. Vashist, "Design and modeling of MEMS-based trace-level moisture measurement system for GIS applications in smart grid environment", IEEE Sensors Journal, vol. 17, no. 23, pp. 7758-7766, 2017.

[32] CIGRE WG 23-10, "SF6 Recycling Guide," ELECTRA 173 (1997), 43-69.

[33] CIGRE WG 23-02, "Guide for SF6 gas mixtures," CIGRE technical brochure 163, August 2000, available via http://www.cigre-sc23.org/publications

[34] IEEE Standard 1125-1993, "IEEE Guide for Moisture Measurement and Control in SF6 Gas-Insulated Equipment", IEEE Power and Energy Society, 2004.

[35] IEC60480-2004, "Guidelines for the checking and treatment of sulfur hexafluoride (SF6) taken from electrical equipment and specification for its re-use," International Electrotechnical Commission, 2004.

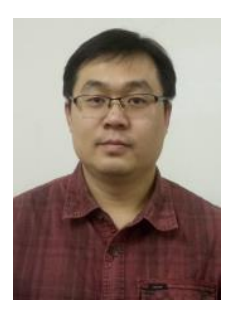

Baojun Qu is a $\mathrm{PhD}$ student at Province-Ministry Joint Key Laboratory of EFEAR, Hebei University of Technology, China. His research interests include sensor design and measurement systems.

Dr Qingxin Yang is a full Professor at Tianjin Key Laboratory of AEEET, Tianjin Polytechnic University, China. He is currently the President of Tianjin Polytechnic University,

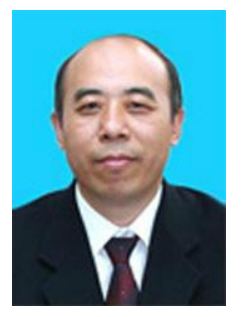

China. His research interests include magnetics measurement and control.

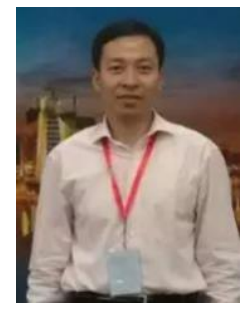

Dr Yongjian Li (IEEE member) is a full Professor at the Province-Ministry Joint Key Laboratory of EFEAR, Hebei University of Technology, China. He is currently the vice-dean at School of Electrical Engineering, Hebei University of Technology, China. His research interests include magnetics measurement and control.

Dr. Reza Malekian (M'12-SM'17) is currently an Associate

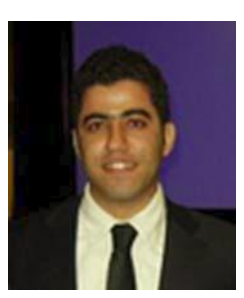
Professor and Head of Advanced Sensor Network Research Group with the Department of Electrical, Electronic, and Computer Engineering, University of Pretoria, Pretoria, South Africa. His current research interests include advanced sensor networks, Internet of Things, and mobile communications. Dr. Malekian is the Joint Chapter Chair of IEEE Signal Processing and Communications Societiess, South Africa section. He is also a Chartered Engineer and a Professional Member of the British Computer Society. He is an associate editor for IEEE Internet of Things Journal and IEEE Transactions on Intelligent

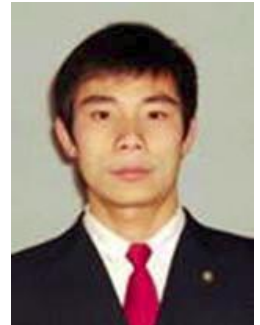
Transportation Systems..

Dr. Zhixiong Li (M'16) received his $\mathrm{PhD}$ in Transportation Engineering from Wuhan University of Technology, China. Currently he is an Assistant Professor with China University of Mining and Technology, China, and a researcher with University of Wollongong, Australia. His research interests include mechanical system modeling and control. He is an editor of IEEE ITS Magazine. 